\title{
Sensory approaches in mental health: Contemporary occupation-based practice or a redundant medical approach?
}

\section{Tawanda Machingura, Chris Lloyd}

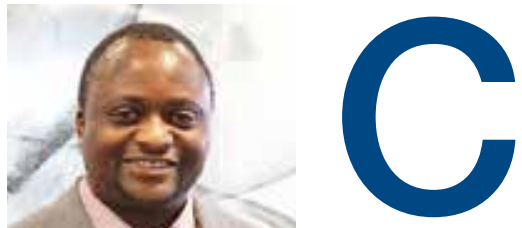

ompetition of ideas is essential for the advancement of a profession. It is essential that theorists, researchers, philosophers and others involved in the advancement of the profession not only focus on doing good (beneficence) but also focus on not doing harm (maleficence). When exponents of a theory or practice are only focused on advancing their theory or practice while not responding to critics, we would argue that this approach fails the integrity test, although the intention is beneficence. Likewise, when critics of a theory or practice do not apply the same rigorous review to their own theories or practices, they too fail the integrity test, although their intention is maleficence. Instead, we argue that the integrity test is met when exponents of a theory are open to and respond to critics and when critics apply the same rigour of review to their own theories and practices. In the profession of occupational therapy, it is not unusual for emerging theories or practices to have supporters and opponents. For example, occupational science had its supporters and opponents who questioned and defended its usefulness in the early 1990s (Pierce, 2012). Similarly the emergence of sensory approaches as a key approach in mental health occupational therapy has stimulated debate within the profession, with critics arguing that they are not occupation focused.

Chris Lloyd Adjunct senior research fellow, Griffith University, Australia

\section{Sensory approaches}

Sensory approaches are based on a theory developed by Ayres (1972), known as sensory integration. Sensory integration is the neurological process that enables people to make sense of the world by receiving, registering, modulating, organising, and interpreting information that the brain receives from senses (Ayres, 1972). Dysfunction in sensory integration explains functional problems observed in some people who may have trouble learning new skills, organising themselves, regulating their attention, participating in work, school or play activities, and engaging in positive social experiences.

Exponents of sensory approaches in clinical practice have reported them as generally effective (Champagne, 2004; Champagne and Stromberg, 2004; Champagne, 2011; Sutton and Nicholson, 2011; Chalmers et al, 2012; Novak et al, 2012; Sutton et al, 2013; Lloyd et al, 2014; Scanlan and Novak, 2015). Sensory approaches are currently widely used in both inpatient and community mental health settings, particularly for decreasing distress during crisis situations. Typically, clinicians make use of sensory environments, such as a sensory room, sensory equipment such as a weighted blanket and sensory activities such as rocking, to regulate these individuals' sensory experiences and optimise physiological and emotional wellbeing. In mental health care, providing opportunities for people experiencing acute symptoms to regulate their own emotional and behavioural responses is seen as a promising intervention (Sutton and Nicholson, 2011). This type of sensory intervention is known as sensory modulation, which aims to achieve a calm or alert arousal state (Sutton and Nicholson, 2011; Champagne and Koomar, 2012; Sutton et al, 2013; Lloyd et al, 2014; Scanlan and Novak, 2015).

Despite the growing practice-based evidence suggesting that sensory approaches are effective (Sutton and Nicholson, 2011; Champagne and Koomar, 2012; Sutton et al, 2013; Lloyd et al, 2014), critics within the field of occupational therapy have argued that such practices are not 'occupation focused/based' and therefore not in touch with contemporary occupational therapy practice. They also argue that the evidence on effectiveness have been less rigorous, such as singlesubject designs, case studies, and quasi-experimental designs (Lloyd et al, 2014; Scanlan and Novak, 2015).

\section{Occupation}

In occupational therapy, occupation is used in therapy in two key ways: as a means and an ends. This means that occupation can be used in therapy with clients as the means to achieve their goals and/or used as an ends to the client's goals being to participate in particular occupations. We argue that when using sensory approaches, occupation should be used as a means and an ends. Second, we suggest that occupation should be the focus when using sensory approaches rather than remediation.

\section{Shifting focus from remediation to occupation}

The goals of intervention should be aimed at enhancing the person's ability to participate in the 
daily occupations which they need to, want to, or are expected to do (WFOT, 2012). Sensory approaches undoubtedly utilise a remediation approach through the provision of various sensory experiences. This typically involves one-to-one direct intervention in an environment that has a variety of specialised equipment such as a sensory room. When examining the exponents and critics viewpoints, it is clear that good practice principles are needed to guide current practice.

Based on our review of the literature and available studies as summarised in a review by Scanlan and Novak (2015) and our own practice experience, we suggest that the focus be shifted to adapting the person's environment in ways that will facilitate the person's ability to participate in their daily occupations as below.

\section{Best practice principles}

- Focus first and foremost on the occupations identified by the person that are of concern to them. This will help to maintain an occupational focus.

- Assess the transactions between person, environment and occupation factors affecting occupational performance and determine the need for intervention and appropriateness of use of sensory approaches. Assess sensory profile or preferences and facilitate self-awareness through provision of education to the person and their families, carers, significant others and other team members. This helps to increase self-awareness and uptake of the approach. The

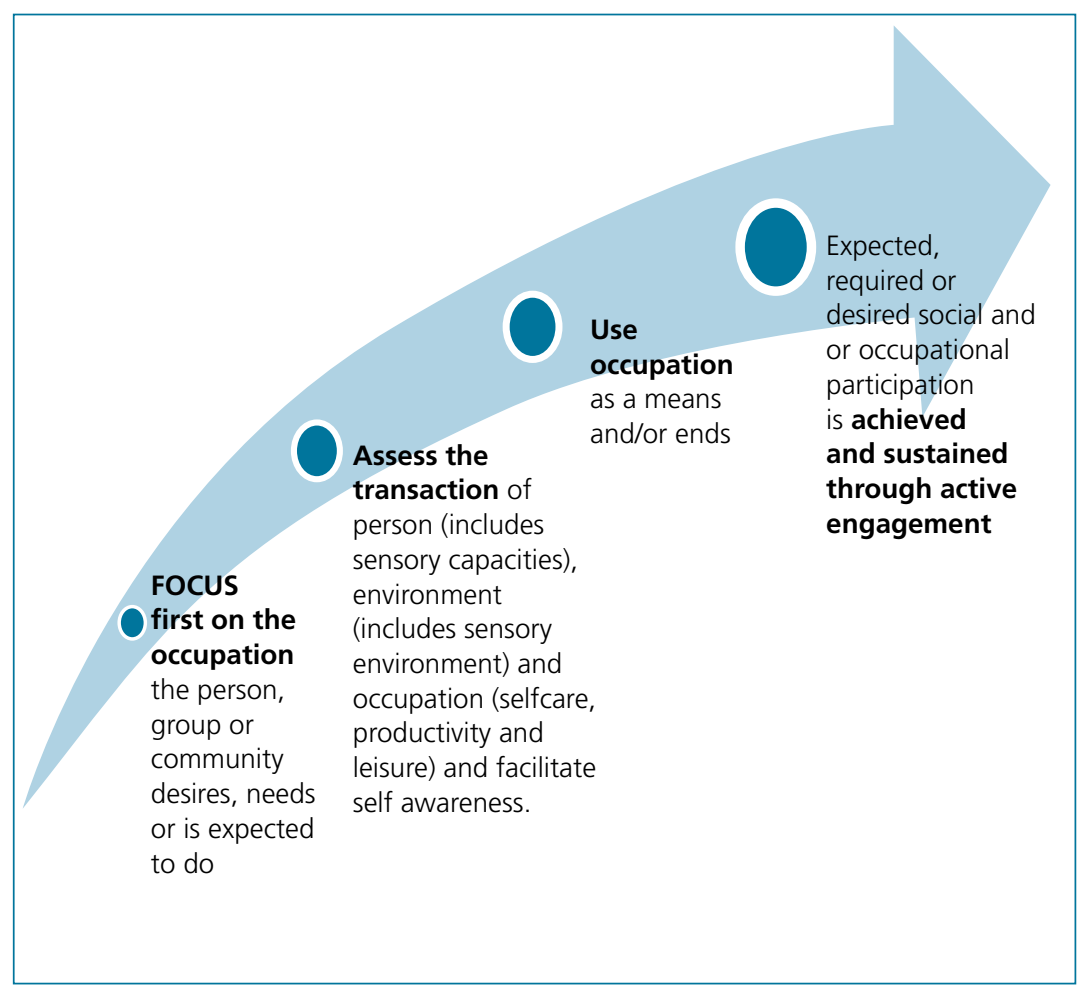

Figure 1. Visual interpretation of the best practice principles client should be empowered, and education plays a major role in being able to achieve this objective.

- Use occupation as a means (occupation is used as the intervention medium to achieve a client's goals) and/or ends (performance in occupations is the goal of intervention). Actively engage and collaborate with client throughout the process.

\section{Conclusions}

Exponents view sensory approaches as evidencedbased emerging practice; critics argue the practice is not occupation-focused and evidence is only emerging. Occupational therapists should maintain an occupation focus and shift their focus from remediation to occupation. IJTR

Ayres AJ . Sensory integration and the child. Los Angeles: Western Psychological Services; 1979.

Chalmers A, Harrison S, Mollison K, Molloy N, Gray K. Establishing sensory-based approaches in mental health inpatient care: a multidisciplinary approach. Australas Psychiatry. 2012;20(1):3539. https://doi.org/10.1177/1039856211430146

Champagne T. The influence of posttraumatic stress disorder, depression, and sensory processing patterns on occupational engagement: a case study. Work. 2011;38(1):67-75. https:// doi.org/10.3233/WOR-2011-1105

Champagne T, Frederick D. Sensory processing research advances in mental health: implications for occupational therapy. OT Practice. 2011;16(10):7-8.

Champagne T, Koomar J. Evaluating sensory processing in mental health occupational therapy practice. OT Practice. 2012;17(5): CE-1-CE-8.

Champagne T, Stromberg N. Sensory approaches in inpatient psychiatric settings: innovative alternatives to seclusion and restraint. J Psychosoc Nurs Ment Health Serv. 2004;42(9):34-44. https://doi.org/10.1002/oti.97

Lloyd C, King R, Machingura T. An investigation into the effectiveness of sensory modulation in reducing seclusion within an acute mental health unit. Adv Mental Health. 2014;12(2): 93-100. https://doi.org/10.1080/18374905.2014 .11081887

Novak T, Scanlan J, McCaul D, MacDonald N, Clarke T. Pilot study of a sensory room in an acute inpatient psychiatric unit. Australas Psychiatry. 2012;20(5):401-406. https://doi. org/10.1177/1039856212459585

Pierce D. The 2011 Ruth Zemke lecture in occupational science: Promise. J Occup Sci. 2012;19(4):298-311.

Scanlan JN, Novak T. Sensory approaches in mental health: A scoping review. Aust Occup Ther J. 2015;62(5):277-285. https://doi.org/10.1111/1440-1630.12224

Sutton D, Nicholson E. Sensory modulation in acute mental health wards: A qualitative study of staff and service user perspectives. Auckland, New Zealand: Te Pou o Te Whakaaro Nui; 2011 [cited 2017 August 23]. Available from: http:// www.tepou.co.nz/uploads/files/resource-assets/sensory_ modulation_in_acute_mental_health_wards.pdf

Sutton D, Wilson M, Van Kessel K, Vanderpyl J. Optimising arousal to manage aggression: a pilot study of sensory modulation. Int J Ment Health Nurs. 2013;22(6):500-511.

World Federation of Occupational Therapy. Definition of occupational therapy. 2012 [cited 2017 August 23]. Available from: http://www.wfot.org/AboutUs/AboutOccupationalTherapy/ DefinitionofOccupationalTherapy.aspx 